\title{
Algebraic Characterizations of Unitary Linear Quantum Cellular Automata
}

\author{
Pablo Arrighi \\ IMAG Laboratories \& University of Grenoble, \\ 46 Avenue Félix Viallet, 38031 Grenoble Cedex, France. \\ pablo.arrighi@imag.fr
}

\begin{abstract}
We provide algebraic criteria for the unitarity of linear quantum cellular automata, i.e. one dimensional quantum cellular automata. We derive these both by direct combinatorial arguments, and by adding constraints into the model which do not change the quantum cellular automata's computational power. The configurations we consider have finite but unbounded size.
\end{abstract}

\section{Motivations}

One could say that the central question in theoretical computer science is 'What are the resources necessary for computation, or information processing?'. Ultimately this question is dictated by the physical laws which surround us. Quantum computation science has risen from this basic idea. It considers computers as physical, and hence possibly quantum systems. At the theoretical level it was demonstrated for instance that polynomial-time integer factorization is possible with such systems, as well as the search for an element in a unordered list of size $n$ in time $\Theta(\sqrt{n})$.

Cellular automata (CA) are arrays of cells, each of which may take one in a finite number of possible state. These evolve in discrete time steps according to a global evolution $\Delta$ - which itself arises from the application of a local transition function $\delta$, synchronously and homogeneously across space. A popular example is Conway's 'Game of Life', a two dimensional CA which has been proven to be universal for computation.

It is clear that CA are themselves physics-like models of computations, as they describe a world of small systems interacting locally, according to translationinvariant laws. Therefore it seems natural to study their quantum extensions. Moreover no classical control is required in such models, since computation arises as an emergent behaviour of the quantum cells' interaction. This is a key advantage to have as it reduces the need for environment interaction, and hence may reduce decoherence, the principal obstacle to realizing a quantum computer. For these two reasons Feynman [6], in his seminal paper about quantum computation, has argued that the study of quantum CA may prove an important path to a realistic physical implementation of quantum computers. Another series of legitimate aims is to endow quantum computation with spatio-temporal notions, 
or even to provide a bridge through which computer science notions, such as universality, may contribute to modern theoretical physics. To put it differently such works are a contribution to the understanding of dynamics in discrete, quantum spacetime - but from an idealized, computer-science viewpoint.

Similarly to their classical counterparts linear quantum cellular automata (LQCA) consist of a row of identical, finite dimensional, quantum systems. These evolve in discrete time steps according to a global evolution $\Delta$ - which itself arises from the application of a local transition function $\delta$, homogeneously and synchronously across space. But in order to grant LQCA the status of physically acceptable model of computation, one must ensure that the global evolution $\Delta$ is physically acceptable in a quantum theoretical setting, i.e. one must ensure that $\Delta$ is unitary. Unfortunately this global property is rather non-trivially related to the description of the local transition function $\delta$ - witness of this the abundant literature on reversible cellular automata (RCA), tackling the classical counterpart of this issue. It is actually a very surprising fact that so much has been done to study RCA - when reversibility is not so much of a crucial feature to have in classical computation. A frequently encountered argument states that all consumption-less, zero-heat micro-mechanical device need be reversible. But tracing back the origins of this argument, we find quantum physical considerations once more 9 .

One way to approach this issue is to find a decision procedure, which given $\delta$ tells whether $\Delta$ is unitary. This test should be performed efficiently, so as not to carry any of the complexity of the computation, and be applied to any candidate $\delta$ as a mean to exclude the non-physical ones. Such a strong contribution was indeed achieved by Dürr et al. It does not put an ending point to the problem however, because the number of local transition functions which do indeed induce a unitary global evolution is likely to be rather scarce, as was the case for RCA [1]. Moreover this relatively complicated decision procedure takes an elegant detour via finite automata, but one which does not guide us to understand which $\delta$ 's will eventually yield a physical $\Delta$.

Physicists are used to checking whether an evolution is physically acceptable, but they like to do so algebraically (e.g. $U^{\dagger} U=U U^{\dagger}=\mathbb{I}$ for unitarity, when $U$ is a finite matrix). Much in the same way computer scientists are used to checking whether a program is valid, but like these criteria to be syntactic. When this is not the case, we tend to consider that the definition chosen to formalize the model of computation is in fact too lose. Indeed once universality has been reached, adding more expressiveness does not mean adding more computational power, but only more ways of expressing the same computation. An undesirable excess arises when the syntax proposed by the definition allows the description of non-valid (unrealistic, non-physical) programs, thereby requiring that the user performs non-trivial (non-syntactic) decision procedures to exclude those instances. This is the current state of affairs with LQCA.

Therefore a different, complementary approach is to tighten the definition of linear quantum cellular automata, i.e. to seek for a more restrictive definition whose unitarity may be checked algebraically/syntactically, and yet capable of 
expressing the exact same set of global evolutions as our original definition. In this paper we provide algebraic characterizations of unitary linear quantum cellular automata. We derive these both by direct combinatorial arguments, and by adding constraints into the model which do not change the quantum cellular automata's computational power.

The breakdown of the core of this paper will be given after LQCA are presented formally, in the following section. Our main theorem is stated in the conclusion section, and discussed in comparison to some related approaches.

Notations. Throughout the paper we will denote by $\mathcal{H}_{S}$ the hilbert space whose canonical orthonormal base vectors are identified with the elements of the countable set $S$. E.g. $\mathcal{H}_{\{a a, a b, b a, b b\}}$ is the four dimensional space with canonical orthonormal base $\{|a a\rangle,|a b\rangle,|b a\rangle,|b b\rangle\}$. This means that any vector $\alpha|a a\rangle+\beta|a b\rangle+$ $\gamma|b a\rangle+\delta|b b\rangle$ with $\alpha, \beta, \gamma, \delta \in \mathbb{C}$ belongs to $\mathcal{H}_{\{a a, a b, b a, b b\}}$. Such a vector must be thought of as a superposition of the words $a a, a b, b a, b b$. Moreover the symbol $\mathbf{0}$

is to denote the null vector, not to be confused with $O$ the matrix containing only ones.

\section{The model}

We start with the definition proposed by [14] and [4]. This definition will evolve throughout the paper.

\section{Working definition 1 (LQCA)}

A linear quantum cellular automaton (LQCA) is a 4-tuple $\mathcal{A}=(\Sigma, q, N, \delta)$, where (with $q \Sigma=\{q\} \cup \Sigma$ ):

$-\Sigma$ is a finite set of symbols (i.e. "the alphabet", giving the possible basic states each cell may take);

- $q$ is a symbol such that $q \notin \Sigma$ (i.e. "the quiescent symbol", which may be thought as a special state for empty cells);

- $N$ is a set of $n$ successive signed integers (i.e. "the neighbourhood", telling which cell is next to whom);

- $\delta: \mathcal{H}_{(q \Sigma)^{n}} \rightarrow \mathcal{H}_{q \Sigma}$ is a function from superpositions of $n$ symbols words to superpositions of one symbol words (i.e. "the local transition function", describing the way a cell interacts with its neghbours).

Moreover $\delta$ must verify:

- the quiescent stability condition: $\left.\left[\delta\left|q^{n}\right\rangle\right)=|q\rangle\right]$;

- the no-nullity condition: $\forall w \in(q \Sigma)^{n},[\delta|w\rangle \neq \mathbf{0}]$.

By 'successive' we mean that the number follow each other in unit step, i.e. the neighbourhood is an interval. In the literature these are sometimes referred to as simple neighbourhoods, but it is trivial to simulate non-simple neighbourhoods automata with simple neighbourhoods automata. At this point we need not have a normalization condition such as $\forall w \in(q \Sigma)^{n},[\| \delta|w\rangle \|=1]$. Configurations hold the basic states of an entire row of cells. As we will now formalize ours are finite but unbounded. Note that fixed-sized periodic configurations [13 as well 
as infinite configurations 12 have also been studied, leading to very different results and proof methods (see Section [6 for a discussion).

\section{Definition 1 (finite configurations, interval domains)}

$A$ (finite) configuration $c$ of the quantum cellular automaton $\mathcal{A}=(\Sigma, q, N, \delta)$ is a function $c: \mathbb{Z} \longrightarrow q \Sigma$, with $i \longmapsto c(i)=c_{i}$, such that there exists a (possibly empty) interval $I$ verifying $i \in I \Rightarrow c_{i} \in q \Sigma$ and $i \notin I \Rightarrow c_{i}=q$. Moreover we denote by idom $(c)$ the smallest such interval $I$, referred to as the interval domain of $c$. Then the interval $[k+\min (N), l+\max (N)]$ is denoted extidom(c) and referred to as the extended interval domain of $c$. For the all-quiescent configuration $c=\ldots q q q \ldots$ we have idom $(c)=\emptyset$, and we let extidom $(c)=\emptyset$ also. Finally the set of all finite configurations is denoted $\mathcal{C}_{f}$, whilst the set of configurations having interval domains comprised within an interval $J$ is denoted $\mathcal{C}_{f}^{J}$.

\section{Definition 2 (indexing conventions)}

Given a configuration $c$ of the quantum cellular automaton $\mathcal{A}=(\Sigma, q, N, \delta)$, we denote by $c_{k \ldots l}$ the word $c_{k} \cdot \ldots \cdot c_{l}$ if $k \leq l$, and the empty word $\varepsilon$ otherwise. Thus in either case $c_{k \ldots l} \in(q \Sigma)^{*}$. Moreover we denote by $c_{i+N}$ the word $c_{i+\min (N) \ldots i+\max (N)}$, and $c_{i+\tilde{N}}$ the word $c_{i+\min (N) \ldots i+\max (N)-1}$. Therefore we have $c_{i+N} \in(q \Sigma)^{n}$ and $c_{i+\tilde{N}} \in(q \Sigma)^{n-1}$, respectively.

Whilst configurations hold the basic states of an entire row of cells, and hence denote the possible basic states of the entire LQCA, the global state of a LQCA may well turn out to be a superposition of these. The following definition works because $\mathcal{C}_{f}$ is a countably infinite set.

\section{Definition 3 (superpositions of finite configurations)}

$A$ superposition of configurations of the quantum cellular automaton $\mathcal{A}=(\Sigma, q, N, \delta)$ is a normalized element of $\mathcal{H}_{\mathcal{C}_{f}}$, the Hilbert space of configurations.

\section{Definition 4 (global evolution)}

The global evolution of the quantum cellular automaton $\mathcal{A}=(\Sigma, q, N, \delta)$ is the linear operation defined by linear extension of its action upon the canonical orthonormal basis, as follows:

$$
\begin{aligned}
& \Delta: \mathcal{H}_{\mathcal{C}_{f}} \rightarrow \mathcal{H}_{\mathcal{C}_{f}} \\
&|c\rangle \mapsto \Delta|c\rangle \\
& \Delta|c\rangle=\bigotimes_{i \in \mathbb{Z}} \delta\left|c_{i+N}\right\rangle
\end{aligned}
$$

The postulates of quantum theory impose that the global evolution should be unitary.

\section{Definition 5 (Unitarity)}

The global evolution $\Delta$ of the quantum cellular automaton $\mathcal{A}=(\Sigma, q, N, \delta)$ is said to be (finite) unitary if and only if $\left\{\Delta|c\rangle \mid c \in \mathcal{C}_{f}\right\}$ is an orthonormal basis of $\mathcal{H}_{\mathcal{C}_{f}}$. 
The next three lemmas are known facts in linear algebra which will prove useful later on.

\section{Lemma 1 (Norm-preservedness, norm of rows)}

Let $\Delta: \mathcal{H}_{S} \rightarrow \mathcal{H}_{S}$ be a linear operator over a Hilbert space $\mathcal{H}_{S}$ having canonical orthonormal basis $\{|c\rangle\}_{c \in S}$. Suppose the following two conditions are fulfilled simultaneously:

-(i) $\forall c \in S,[\| \Delta|c\rangle||=1]$;

-(ii) $\forall c, c^{\prime} \in S,\left[\left\langle c^{\prime}\left|\Delta^{\dagger} \Delta\right| c\right\rangle \neq 0 \Leftrightarrow c=c^{\prime}\right]$;

Then $\forall r \in S,\left[0 \leq \| \Delta^{\dagger}|r\rangle \| \leq 1\right]$.

Proof: Conditions (i) and (ii) express the fact that $\Delta$ is norm-preserving. As a consequence for all $r \in S$ we have $\left.\| \Delta^{\dagger}|r\rangle\left|\|=\| \Delta \Delta^{\dagger}\right| r\right\rangle \|$. Moreover we have by definition $\| \Delta^{\dagger}|r\rangle||^{2}=\left|\left\langle r\left|\Delta \Delta^{\dagger}\right| r\right\rangle\right|$. But the latter $\left(\left\langle r\left|\Delta \Delta^{\dagger}\right| r\right\rangle\right)$ is a projection of the former $\left(\Delta \Delta^{\dagger}|r\rangle\right)$ over a unit vector $(|r\rangle)$, and hence $\left|\left\langle r\left|\Delta \Delta^{\dagger}\right| r\right\rangle\right| \leq \| \Delta \Delta^{\dagger}|r\rangle||$. Therefore $\| \Delta^{\dagger}|r\rangle\left\|^{2} \leq\right\| \Delta^{\dagger}|r\rangle \|$ and so $\| \Delta^{\dagger}|r\rangle \| \leq 1$.

As a corollary we have:

\section{Lemma 2 (Norm-preservedness, finite spaces)}

Let $\Delta: \mathcal{H}_{S} \rightarrow \mathcal{H}_{S}$ be a linear operator over a Hilbert space $\mathcal{H}_{S}$ having canonical orthonormal basis $\{|c\rangle\}_{c \in S}$. Let $T$ be a finite subset of $S$. Suppose the following three conditions are fulfilled simultaneously:

-(i) $\forall c \in S,[\| \Delta|c\rangle||=1]$;

-(ii) $\forall c, c^{\prime} \in S,\left[\left\langle c^{\prime}\left|\Delta^{\dagger} \Delta\right| c\right\rangle \neq 0 \Leftrightarrow c=c^{\prime}\right]$;

-(iii) $\left[\sum_{r \in T} \| \Delta^{\dagger}|r\rangle \|^{2}=|T|\right]$.

Then we have $\forall r \in T,\left[\| \Delta^{\dagger}|r\rangle \|=1\right]$.

\section{Lemma 3 (Unitarity from unit rows)}

Let $\Delta: \mathcal{H}_{S} \rightarrow \mathcal{H}_{S}$ be a linear operator over a Hilbert space $\mathcal{H}_{S}$ having canonical orthonormal basis $\{|c\rangle\}_{c \in S}$. Suppose the following three conditions are fulfilled simultaneously:

-(i) $\forall c \in S,[\| \Delta|c\rangle||=1]$;

-(ii) $\forall c, c^{\prime} \in S,\left[\left\langle c^{\prime}\left|\Delta^{\dagger} \Delta\right| c\right\rangle \neq 0 \Leftrightarrow c=c^{\prime}\right]$;

-(iii) $\forall r \in S,\left[\| \Delta^{\dagger}|r\rangle||=1\right]$.

Then $\Delta$ is unitary.

Proof: Conditions (i) and (ii) express the fact that $\{\Delta|c\rangle \mid c \in S\}$ is an orthonormal set, i.e. that $\Delta$ is norm-preserving. Condition (iii) expresses the fact that for all $r \in S, \Delta^{\dagger}|r\rangle$ has unit norm. As a consequence $\Delta \Delta^{\dagger}|r\rangle$ has unit norm on the one hand, and $\left\langle r\left|\Delta \Delta^{\dagger}\right| r\right\rangle=\| \Delta^{\dagger}|r\rangle \|=1$ on the other hand. Therefore $\alpha \Delta \Delta^{\dagger}|r\rangle=|r\rangle$, with $\alpha$ a root of unity. Since $\Delta \Delta^{\dagger}$ is positive, this $\alpha$ is just 1 . Let $\Delta^{\dagger}|r\rangle=\sum \beta_{c}|c\rangle$. Then $|r\rangle=\sum \beta_{c} \Delta|c\rangle$, in other words each of the canonical orthonormal basis vectors $|r\rangle$ may be expressed as a linear combination of columns $\{\Delta|c\rangle \mid c \in S\}$. Therefore the columns form themselves an orthonormal basis.

Next we will examine each of the following conditions in turn: 
-(i) the columns of $\Delta$ have unit norm (section 3);

-(ii) the columns of $\Delta$ are orthogonal (section 4 );

-(iii) the rows of $\Delta$ have unit norm (section 5 ).

\section{Unit columns}

The next two lemmas are simple facts from [4].

Lemma 4 (Norm of a column) Let $\Delta$ denote the global evolution of the quantum cellular automaton $\mathcal{A}=(\Sigma, q, N, \delta)$. We have that

$$
\forall c \in \mathcal{C}_{f}, \quad\left[\| \Delta|c\rangle||=\prod_{i \in \mathbb{Z}} \| \delta\left|c_{i+N}\right\rangle||\right]
$$

Proof. The norm of a tensor product of vectors is the product of the norms of the vectors.

Lemma 5 ( Expressiveness of normalized $\delta$ ) Let $\Delta$ denote the global evolution of the quantum cellular automaton $\mathcal{A}=(\Sigma, q, N, \delta)$. Let $\Delta^{\prime}$ denote the global evolution of the quantum cellular automaton $\mathcal{A}^{\prime}=\left(\Sigma, q, N, \delta^{\prime}\right)$, with $\delta^{\prime}$ such that $\forall w \in(q \Sigma)^{n},\left[\delta^{\prime}|w\rangle=\delta|w\rangle / \| \delta|w\rangle||\right]$. Suppose that the columns of $\Delta$ have unit norm. Then we have $\Delta=\Delta^{\prime}$.

Proof. For all $c$ in $\mathcal{C}_{f}$ we have

$$
\begin{aligned}
\Delta|c\rangle & =\bigotimes_{i \in \mathbb{Z}} \delta\left|c_{i+N}\right\rangle=\bigotimes_{i \in \mathbb{Z}} \| \delta\left|c_{i+N}\right\rangle|| \cdot \delta^{\prime}\left|c_{i+N}\right\rangle \\
& =\prod_{i \in \mathbb{Z}} \| \delta\left|c_{i+N}\right\rangle|| \cdot \bigotimes_{i \in \mathbb{Z}} \delta^{\prime}\left|c_{i+N}\right\rangle \\
& =\| \Delta|c\rangle|| \cdot \bigotimes_{i \in \mathbb{Z}} \delta^{\prime}\left|c_{i+N}\right\rangle=\Delta^{\prime}|c\rangle
\end{aligned}
$$

Our approach is to change the actual definition of LQCA as a consequence.

\section{Working definition 2 (LQCA)}

A linear quantum cellular automaton (LQCA) is a 4-tuple $\mathcal{A}=(\Sigma, q, N, \delta)$, where:

- $\Sigma$ is a finite set of symbols ("the alphabet");

- $q$ is a symbol such that $q \notin \Sigma$ ("the quiescent symbol");

- $N$ is a set of $n$ successive signed integers ("the neighboorhood");

- $\delta: \mathcal{H}_{(q \Sigma)^{n}} \rightarrow \mathcal{H}_{q \Sigma}$ is a function from superpositions of $n$ symbols words to superpositions of one symbol words ("the local transition function").

Moreover $\delta$ must verify the following two properties:

- the quiescent stability condition: $\left.\left[\delta\left|q^{n}\right\rangle\right)=|q\rangle\right]$.

- the normalization condition:

$\forall w \in(q \Sigma)^{n},[\| \delta|w\rangle \mid \|=1]$. 
This modified definition of LQCA choses to impose that $\forall w \in(q \Sigma)^{n}$, [ $[|\delta| w\rangle \|=$ $1]$, i.e. that the local transition function $\delta$ is normalized. Then the fact that the columns of $\Delta$ have unit norm follows straight from lemma 4 We are strongly justified to place this algebraic, almost syntactic restriction straight into the definition of LQCA because:

- The alternative is to have various non-normalized states compensating each other non-locally, which from a physical point of view is somewhat disturbing;

- It saves us from having to employ more elaborate techniques to check that columns have unit norms [4 [7, such as applying least path algorithm to the associated de Bruijn graphs of the quantum cellular automata etc. Although very elegant these tend to render quantum cellular automata much more oblivious as a model of computation;

- The modification made has absolutely no cost in terms expressiveness, as demonstrated in Lemma 5

\section{Orthogonality of columns}

Having checked that the columns of the global evolution matrix $\Delta$ have unit norm, we now turn to the problem of deciding whether these columns are mutually orhogonal. First we need a definition.

Definition 6 ( $A$-matrix) Consider a linear quantum cellular automaton $\mathcal{A}=$ $(\Sigma, q, N, \delta)$. We call $A=\left[A_{x y}^{\sigma}\right]$ with $x, y \in(q \Sigma)^{n-1}$ and $\sigma \in(q \Sigma)$, the matrix (tensor) such that $A_{x y}=\sum_{\sigma} A_{x y}^{\sigma}|\sigma\rangle$ equals $\delta|w\rangle$ if we have both $x=w_{1 \ldots n-1}$ and $y=w_{2 \ldots n}$ for some $w \in(q \Sigma)^{n}$, otherwise it is the null vector.

For instance consider the sample rule: $\delta|000\rangle=|0\rangle, \delta|001\rangle=|1\rangle, \delta|010\rangle=|1\rangle$, $\delta|011\rangle=|0\rangle, \delta|100\rangle=|0\rangle, \delta|101\rangle=|1\rangle, \delta|110\rangle=|1\rangle, \delta|111\rangle=|0\rangle$.

Then the $A$-matrix of the sample rule is:

$$
\left(\begin{array}{cccc}
|0\rangle & \mathbf{0} & |0\rangle & \mathbf{0} \\
|1\rangle & \mathbf{0} & |1\rangle & \mathbf{0} \\
\mathbf{0} & |1\rangle & \mathbf{0} & |1\rangle \\
\mathbf{0} & |0\rangle & \mathbf{0} & |0\rangle
\end{array}\right) .
$$

E.g. since 01 and 00 do not "follow each other" the entry $\langle 00|A| 01\rangle$ holds the null vector. On the other hand 01 and 10 do overlap correctly to form the neighbourhood 010, for which $\delta|010\rangle=|1\rangle$, hence $\langle 10|A| 01\rangle=|1\rangle$.

How can we check that all columns of some global evolution are mutually orthogonal, when there is infinitely many of them? Our next proposition is crucial in that respect, as it shows why the problem which might seem to be of an infinite (undecidable) nature is indeed of a finite (decidable) nature.

\section{Proposition 1 (Finite columns checks)}

This result refers to working definition 2

Consider the global evolution $\Delta$ of a quantum cellular automaton $\mathcal{A}=(\Sigma, q, N, \delta)$.

Let $s$ be equal to $|q \Sigma|^{2 n-2}-1$ and I be the interval $[0, s]$. The columns $\{\Delta|c\rangle \mid c \in$ $\left.\mathcal{C}_{f}\right\}$ are orthogonal if and only if the columns $\left\{\Delta|c\rangle \mid c \in \mathcal{C}_{f}^{I}\right\}$ are orthogonal. 
(See appendix A.1 for a detailed proof.)

We now give our algebraic condition upon $\delta$ ensuring that the columns of $\Delta$ are mutually orthogonal.

\section{Proposition 2 (Column test)}

This result refers to working definition 2

Consider the global evolution $\Delta$ of a quantum cellular automaton $\mathcal{A}=(\Sigma, q, N, \delta)$.

Let $s=|q \Sigma|^{2 n-2}-1$. The columns $\left\{\Delta|c\rangle \mid c \in \mathcal{C}_{f}\right\}$ are orthogonal if and only if $\forall x, x^{\prime} \in(q \Sigma)^{n-1}$ :

$$
\left[\left\langle x x^{\prime}\left|M^{s}\right| q^{n-1} q^{n-1}\right\rangle\left\langle q^{n-1} q^{n-1}\left|M^{s}\right| x x^{\prime}\right\rangle \neq 0 \Leftrightarrow\left(x=x^{\prime}\right)\right]
$$

with $M=\left[M_{x x^{\prime}, y y^{\prime}}\right], M_{x x^{\prime}, y y^{\prime}}=\left|\sum_{\sigma} A_{x^{\prime} y^{\prime}}^{\sigma *} A_{x y}^{\sigma}\right|^{2}$, A the A-tensor of the LQCA.

(See appendix A.2 for a detailed proof.)

We believe that the obtention of algebraic conditions constituted a necessary step in order to be able to take further the analysis of this model. The algebraic proofs of these conditions give them a physical meaning which shortcuts the graph-theoretical detour, which is particularly useful since quantum theory people tend to reason in terms of linear algebra rather than graph theory. They also master the corresponding numerical tools better, Proposition 2 makes it easy to check for column orthonormality through any software tool which does matrix multiplication.

Still there remains some space for improvement, for instance because Proposition 2 is phrased in terms of the somewhat bizarre $A$-tensor of $\mathcal{A}$, rather than just $\delta$. Fortunately this first point can be fixed using the quantum equivalent of a simplifying classical result [8] [1] [3]

\section{Lemma 6 (Expressivity of size two $\delta$ )}

This result refers to working definition 2

Consider a linear quantum cellular automaton $\mathcal{A}^{\prime}=\left(\Sigma, q, N, \delta^{\prime}\right)$ and its global evolution $\Delta^{\prime}$. One can always construct a linear quantum cellular automaton $\mathcal{A}=\left(\Sigma^{n-1}, q^{n-1},\{0,1\}, \delta\right)$ such that its global evolution $\Delta$ equals $\Delta^{\prime}$. Then the $A$-tensor of $\mathcal{A}$ is just $\delta=\left[\delta_{x y}^{\sigma}\right]$, with $x, y, \sigma \in \Sigma^{n-1}$.

Proof. We let, for all $x, y \in \Sigma^{n-1}$

$$
\delta\left|x_{1} \ldots x_{n-1} y_{1} \ldots y_{n-1}\right\rangle \equiv \bigotimes_{i \in[1, n-1]} \delta^{\prime}\left|x_{i} \ldots x_{n-1} y_{1} \ldots y_{i}\right\rangle
$$

The rest follows from the definitions.

Note that the groups of $n-1$ cells constructed in this lemma bear some resemblance with the reduced neighbourhoods $\tilde{N}$ constructed by Dür et al. - except they do not overlap, which consequently saves us from using De Buijn graphs and the like. This suggests that in spite of its apparent simplicity this trick is probably just the right way to enumerate/construct LQCA.

We change the definition of LQCA as a consequence. 


\section{Working definition 3 (LQCA)}

A linear quantum cellular automaton $(L Q C A)$ is a 3-tuple $\mathcal{A}=(\Sigma, q, \delta)$, where:

- $\Sigma$ is a finite set of symbols ("the alphabet");

- $q$ is a symbol such that $q \notin \Sigma$ ("the quiescent symbol");

- $\delta: \mathcal{H}_{(q \Sigma)^{2}} \rightarrow \mathcal{H}_{q \Sigma}$ is a function from superpositions of 2 symbols words to superpositions of one symbol words ("the local transition function").

Moreover $\delta$ must verify the following two properties:

- the quiescent stability condition: $[\delta|q q\rangle)=|q\rangle]$.

- the normalization condition:

$\forall w \in(q \Sigma)^{2},[|| \delta|w\rangle||=1]$.

This modified definition of LQCA choses to impose neighbourhoods of size two on top of the normalization condition. For these linear quantum cellular automata we define $\Delta$ as usual with $N=\{0,1\}$. Again we are strongly justified to place this easy restriction straight into the definition of LQCA for it simplifies and makes more intuitive the decision procedure induced by Proposition 2 (the corresponding simplifications are shown in Corollary 1). The modification made comes at absolutely no cost in terms expressiveness, as demonstrated in Lemma 6]

These successive two modifications we have made to our model will be even more asserted by the important simplification they bring to the problem of determining whether a LQCA has unit rows.

\section{Unit rows}

Having checked that the columns of the global evolution matrix $\Delta$ are orthonormal, we now turn to the problem of deciding whether its rows have unit norm.

\section{Proposition 3 (row norm as matrix product)}

This result refers to working definition 3

Consider a quantum cellular automaton $\mathcal{A}=(\Sigma, q, \delta)$ whose global evolution $\Delta$ has orthonormal columns. The squared norm of any row $r$ is given by

$$
\| \Delta^{\dagger}|r\rangle \|^{2}=\lim _{h \rightarrow \infty}\left\langle q\left|N^{(q)^{h}}\left(\prod_{i \in k \ldots l} N^{\left(r_{i}\right)}\right) N^{(q)^{h}}\right| q\right\rangle
$$

where $\left\{N^{(\sigma)}\right\}_{\sigma \in q \Sigma}$ is the set of matrices such that $N^{(\sigma)}=\left[N_{x, y}^{(\sigma)}\right], N_{x, y}^{(\sigma)}=|\langle\sigma|\delta| x y\rangle|^{2}$.

(See appendix $\mathrm{A.3}$ for a detailed proof.)

The following proposition takes advantage of the successive restrictions made in definitions 2 and 3 to bring about a crucial simplification - which makes obsolete a good half of the procedure described in [5]. 


\section{Proposition 4 (Middle segment)}

This result refers to working definition 3

Consider a quantum cellular automaton $\mathcal{A}=(\Sigma, q, \delta)$ whose global evolution $\Delta$ has orthonormal columns. The rows $\left\{\Delta^{\dagger}|r\rangle \mid r \in \mathcal{C}_{f}\right\}$ have unit norm if and only if

$$
\lim _{h \rightarrow \infty}\left\langle q\left|N^{h} O N^{h}\right| q\right\rangle=|q \Sigma|
$$

with $O=\left[1_{x y}\right]$ the matrix with only ones, and $N=\left[N_{x, y}\right], N_{x, y}=|\langle q|\delta| x y\rangle|^{2}$.

(See appendix A.4 for a detailed proof.)

Note that $\lim _{h \rightarrow \infty}\left\langle q\left|N^{h} O N^{h}\right| q\right\rangle=\sum_{r \in \mathcal{C}_{f}^{[0,0]}} \| \Delta^{\dagger}|r\rangle \|^{2}$. Hence we have the following insightful corollary, which comes as the direct analogue of our Proposition 1. Curiously however it will not contribute to our final result.

Proposition 5 (Finite rows check)

This result refers to working definition 3

Consider a quantum cellular automaton $\mathcal{A}=(\Sigma, q, \delta)$ whose global evolution $\Delta$ has orthonormal columns. The rows $\left\{\Delta^{\dagger}|r\rangle \mid r \in \mathcal{C}_{f}\right\}$ have unit norm if and only if the rows $\left\{\Delta^{\dagger}|r\rangle \mid r \in \mathcal{C}_{f}^{[0,0]}\right\}$ have unit norm.

Both results deepen our understanding of the algebraic structure of unitary linear quantum cellular automata. Proposition 4 offers an important simplification along the way to determining whether the global evolution $\Delta$ has unit rows, reducing this to the evaluation of $\vec{l} . O \vec{r}$.

The difficult problem we are left with is that of evaluating the so-called 'border vectors' [5] $\vec{r}=\lim _{h \rightarrow \infty} N^{h}|q\rangle$ and $\vec{l}=\lim _{h \rightarrow \infty} N^{\dagger}|q\rangle$. The following proposition will characterize them uniquely and algebraically. We let $\leq$ denotes the following partial order upon $m \times n$ matrices:

$$
M \leq N \Longleftrightarrow \forall i, j \quad M_{i j} \leq N_{i j}
$$

Column vectors are seen as $m \times 1$ matrices for that matter.

Proposition 6 (Border vectors)

This result refers to working definition 3

Consider a quantum cellular automaton $\mathcal{A}=(\Sigma, q, \delta)$ whose global evolution $\Delta$ has orthonormal columns. The vectors

$$
\vec{r}=\lim _{h \rightarrow \infty} N^{h}|q\rangle \quad \text { and } \quad \vec{l}=\lim _{h \rightarrow \infty} N^{\dagger^{h}}|q\rangle
$$

with $N=\left[N_{x, y}\right], N_{x, y}=|\langle q|\delta| x y\rangle|^{2}$, have only finite entries. They verify

$$
\begin{aligned}
\vec{r} & =\min _{\leq}\left\{v \mid \mathbf{0} \leq v \wedge N v=v \wedge v_{q}=1\right\} \\
\text { and } \quad \vec{l} & =\min _{\leq}\left\{v \mid \mathbf{0} \leq v \wedge v N=v \wedge v_{q}=1\right\} .
\end{aligned}
$$

Moreover the following extra conditions hold:

(i) $\vec{l} \cdot \vec{r}=1$; 
(ii) $\left(\sum_{i} \vec{l}_{i}\right)\left(\sum_{i} \vec{r}_{i}\right) \leq|q \Sigma|$.

(iii) $\forall x \in \Sigma,\left[\vec{l}_{x}=0 \vee \vec{r}_{x}=0\right]$;

(iv) $\forall x, y \in \Sigma,\left[N_{x y} \neq 0 \Rightarrow \vec{l}_{x}=0 \vee \vec{l}_{y}=0\right]$;

Inequality (ii) is saturated if and only if $\Delta$ has unit rows.

(See appendix A.6 for a detailed proof.) This last proposition is highly informative, and in most cases will provide us with an effective way to compute these border vectors, through a spectral decomposition of $N$. When the eigenvalue 1 is degenerate, however, it is unclear to the author whether there exists a definite procedure to performing the minimization.

Taking a step back from the mathematics, we may wonder where these limits $N^{h}, h \rightarrow \infty$ come from 'physically'. Say we wish to calculate the norm of a row $\Delta^{\dagger}|r\rangle$, corresponding to a configuration $r$ with interval domain $I$. Then at some point we need to sum over all the antecedent configurations of $r$ (cf. the beginning of the proof of Proposition 3). Our issue now arises from the fact that although the antecedents of $r$ are in $\mathcal{C}_{f}$, there may be an infinite number of them, and hence they may not be able to restrict to some interval $J$. A good example of this is provided in [5], which we now reproduce for convenience:

Remark 1 Consider QFLIP $=(p, q, \delta)$ with $\delta|q q\rangle=|q\rangle, \delta|q p\rangle=|q\rangle+|p\rangle / \sqrt{2}$, $\delta|p q\rangle=|p\rangle, \delta|p p\rangle=|q\rangle-|p\rangle / \sqrt{2}$. Its corresponding global evolution $\Delta$ is unitary. Let $c^{n}=\ldots q q p^{n} q q \ldots$, with the rightmost $p$ in position 0 . Then $\left\langle c^{1}|\Delta| c^{n}\right\rangle=$ $(1 / \sqrt{2})^{n}$.

Faced with such situations our temptation is again to further restrict the definition of LQCA, with a view to eliminate such scenarios. For instance we may want to add to the Working definition 3 a full stability condition asking that: $\forall w \in \Sigma^{2},[\langle q|\delta| w\rangle=0]$.

This extra 'full stability condition' would ensure that the antecedents $c$ of a configuration $r$ have an interval domain no greater that the extended interval domain of $r$.

\section{Lemma 7 (Full stability unit rows)}

This result refers to working definition 3

Consider a quantum cellular automaton $\mathcal{A}=(\Sigma, q, \delta)$ whose global evolution $\Delta$ has orthonormal columns. Suppose the full stability condition $\forall w \in \Sigma^{2},[\langle q|\delta| w\rangle=$ $0]$. is verified. Then the rows $\left\{\Delta^{\dagger}|r\rangle \mid r \in \mathcal{C}_{b}\right\}$ have unit norm if and only if

$$
\langle q|N O N| q\rangle=|q \Sigma|
$$

with $O=\left[1_{x y}\right]$ the matrix with only ones, and $N=\left[N_{x, y}\right], N_{x, y}=|\langle q|\delta| x y\rangle|^{2}$. 
Proof. First note that under this condition, one has $\forall x \in \Sigma$

$$
\begin{aligned}
& \left\langle x\left|N^{2}\right| q\right\rangle=\sum_{\sigma \in q \Sigma} N_{x \sigma} N_{\sigma q}=\sum_{\sigma \in q \Sigma}|\langle q|\delta| x \sigma\rangle|^{2}|\langle q|\delta| \sigma q\rangle|^{2} \\
& =|\langle q|\delta| x q\rangle|^{2}|\langle q|\delta| q q\rangle|^{2} \quad \text { by the full stability condition } \\
& =|\langle q|\delta| x q\rangle|^{2} \quad \text { by the quiescent stability condition } \\
& =\langle x|N| q\rangle .
\end{aligned}
$$

Moreover we have $\left\langle q\left|N^{2}\right| q\right\rangle=\sum_{\sigma \in q \Sigma} N_{q \sigma} N_{\sigma q}$, which is equal to $N_{q q} N_{q q}=1=$ $\langle q|N| q\rangle$ using the fact that $\forall x \in \Sigma\left[N_{q x} N_{x q}=0\right]$. The contrary would imply $\langle\ldots q q \ldots|\Delta| \ldots q q x q q \ldots\rangle \neq 0$, which is impossible since $\langle q|\delta| q q\rangle=1$ and $\Delta$ is norm-preserving.

As a consequence we have $N^{2}|q\rangle=N|q\rangle$. Symmetrically so for $\langle q| N^{2}=\langle q| N$. The rest follows from Proposition 4 .

Unfortunately it is not clear whether this extra restriction of LQCA implies a loss of expressiveness. In fact may even disqualifies some valid reversible cellular automata from being quantum cellular automata. We leave the question of a simulation as an open problem.

Open problem 1 Consider a linear quantum cellular automaton $\mathcal{A}=(\Sigma, q, \delta)$ following to definition 3 and its global evolution $\Delta$. Is it always possible to construct a linear quantum cellular automaton $\mathcal{A}^{\prime}=\left(\Sigma^{\prime}, q^{\prime}, \delta^{\prime}\right)$ such that $\forall w \in$

$\Sigma^{2},[\langle q|\delta| w\rangle=0]$ and its global evolution $\Delta^{\prime}$ equals $\Delta$ ? (We may want to admit loser notions of efficient simulation.)

In fact one may wonder whether there may not be a way to make any normpreserving LQCA (unit, orthogonal columns) into a unitary LQCA exhibiting the full stability condition. For instance:

Remark 2 Consider XoR $=(\{0,1\}, 0, \delta)$ with $\delta|00\rangle=|0\rangle, \delta|01\rangle=|1\rangle, \delta|10\rangle=$ $|1\rangle, \delta|11\rangle=|0\rangle$. Its corresponding global evolution $\Delta$ is injective in the space of finite configurations (i.e. it is norm-preserving, it has orthonormal columns). But the rule $\delta|11\rangle=|0\rangle$ breaks the full stability condition. Moreover it is not surjective (i.e. its rows are not unit norm) since ...00100 . . does not have any antecedent.

is suitably 'fixed' as in Remark 3

\section{Conclusion}

The following theorem is a synthesis our results.

Theorem 1 (Summary)

This result refers to working definition 3

Consider the global evolution $\Delta$ of a quantum cellular automaton $\mathcal{A}=(\Sigma, q, \delta)$. 
Let $s=|q \Sigma|^{2}-1 . \Delta$ is unitary if and only if $\forall x, x^{\prime} \in(q \Sigma)$ :

$$
\begin{aligned}
& {\left[\left\langle x x^{\prime}\left|M^{s}\right| q q\right\rangle\left\langle q q\left|M^{s}\right| x x^{\prime}\right\rangle \neq 0 \Leftrightarrow\left(x=x^{\prime}\right)\right]} \\
& \text { and } \quad\left(\sum_{i} \vec{l}_{i}\right)\left(\sum_{i} \vec{r}_{i}\right)=|q \Sigma| \\
& \text { with } \vec{r}=\min _{\leq}\left\{v \mid \mathbf{0} \leq v \wedge N v=v \wedge v_{q}=1\right\} ; \\
& \vec{l}=\min _{\leq}\left\{v \mid \mathbf{0} \leq v \wedge v N=v \wedge v_{q}=1\right\} ; \\
& M=\left[M_{x x^{\prime}, y y^{\prime}}\right], M_{x x^{\prime}, y y^{\prime}}=\left|\left\langle x^{\prime} y^{\prime}\left|\delta^{\dagger} \delta\right| x y\right\rangle\right|^{2} ; \\
& N=\left[N_{x, y}\right], N_{x, y}=|\langle q|\delta| x y\rangle|^{2} .
\end{aligned}
$$

We have definitely gone a long way towards the simplification and the algebraization of unitarity criteria for one dimensional quantum cellular automata as defined in [14, 4], [5]. Note that these last two papers do not contain any such synthetic algebraic criteria. Instead they provide several pages long decision procedures, which have many twists and bends.

Certainly we have not gone as far as to reduce LQCA to partitioned quantum cellular automata (PQCA) or quantum cellular automata with Margolus neighbourhood (MQCA) etc., i.e. our global evolution cannot, in general, be decomposed into the application of one small unitary operator homogeneously across space.

Other models of quantum cellular automata do admit such reductions; the problem of deciding unitarity becomes incomparably easier, or even trivial by construction [12. Such crucial differences seem to arise when one considers different spaces of configurations, e.g. finite periodic [13, because these constrain reversibility to be structural, i.e. an essentially local matter. With the space of finite configurations $\mathcal{C}_{f}$ reversibility becomes a global matter, even though the global evolution is defined locally. Analogues of this are well-known in the classical realm already:

Remark 3 Consider XoR' $=(\{q, 0,1\}, q, \delta)$ with $\delta|00\rangle=|0\rangle, \delta|01\rangle=|1\rangle$, $\delta|10\rangle=|1\rangle, \delta|11\rangle=|0\rangle, \delta|q 0\rangle=|q\rangle, \delta|q 1\rangle=|q\rangle, \delta|0 q\rangle=|0\rangle, \delta|1 q\rangle=|1\rangle$. Its corresponding global evolution $\Delta$ is bijective in the space of finite configurations (i.e. it is unitary), but the global evolution cannot be reversed by a cellular automata. Moreover $\Delta$ is not reversible in the space of infinite configurations $(\ldots 00 \ldots$ may have antecedents either ...00 . . or ...11 ..).

One may argue that PQCA models are enough, since they simulate the quantum Turing machine. If we are interested in 'intrinsic universality' however, and want to consider the above example as 'physical' then these are not be enough. A local transition $\delta$ which is unitary in the space of infinite configurations is also unitary in the space if finite configurations, but the reciprocal statement is not true. In this sense there seems to be a loss of expressiveness when restricting to PQCA models. 
Note that this research constitutes a first step in the quest for the identification of the one particular unitary LQCA known to simulate all other LQCA efficiently. Obviously as we restrict the number of one dimensional quantum cellular automata to be considered to a well-behaved subclass, the quest for such an 'intrinsically universal' one dimensional quantum cellular automata must become easier. Moreover our approach may eventually open this (well-advertised in the literature) open problem to an algebraic analysis.

Note also that it is undecidable whether local transition function $\delta$ induces a unitary global evolution $\Delta$ as soon as the quantum cellular automata is two dimensional. But again this does not have to be the end of it: nothing prevents that there should be a canonical definition of two dimensional quantum cellular automata, easily checked for unitarity, and yet capable of expressing the exact same set of global evolutions. For this purpose algebraic criteria ought to be easier to generalize to higher dimensions.

\section{Acknowledgments}

P.J.A would like to thank Shu Yan Chan, Christoph Dürr, Simon Perdrix, and Estrella Sicardi for a number of insightful conversations, and acknowledges the direct help of Thierry Gallay in proving proposition [6 This research was initiated as an Assistant Professor in Montevideo, Uruguay, at the Institute of Physics of the Engineering Faculty and concluded as a CNRS post-doc in Grenoble, France, at the Leibniz Laboratory. Towards both institutions the author is greatly indebted of a very pleasant stay. Many thanks in particular to Gonzalo Abal, Arturo Lezama, Alejandro Romanelli, Ricardo Siri and above all Philippe Jorrand for offering me these excellent working conditions.

\section{A Proofs of Propositions 16}

\section{A.1 Proof of Proposition 1 ,}

Consider $c \neq c^{\prime}$ such that $\Delta|c\rangle$ is not orthogonal to $\Delta\left|c^{\prime}\right\rangle$. Since there are $|q \Sigma|^{2 n-2}$ possible pairs of $(q \Sigma)^{n-1}$ subwords, there must exist $k, l \in \mathbb{Z}$ with $0 \leq l-k \leq s$ such that $c_{k \ldots k+n-2}=c_{l \ldots l+n-2}$ and $c_{k \ldots k+n-2}^{\prime}=c_{l \ldots l+n-2}^{\prime}$. We now construct $d=c_{\infty \ldots k+n-2} \cdot c_{l+n-1 \ldots \infty}$ and $d^{\prime}=c_{\infty \ldots k+n-2}^{\prime} \cdot c_{l+n-1 \ldots \infty}^{\prime}$, and notice that

$$
\begin{aligned}
\left(\Delta|d\rangle, \Delta\left|d^{\prime}\right\rangle\right) & =\prod_{i \notin] k+n-2, l+n-1[}\left(\delta\left|c_{i+N}^{\prime}\right\rangle, \delta\left|c_{i+N}\right\rangle\right) \neq 0 \\
\text { since } \quad\left(\Delta|c\rangle, \Delta\left|c^{\prime}\right\rangle\right) & =\prod_{i}\left(\delta\left|c_{i+N}^{\prime}\right\rangle, \delta\left|c_{i+N}\right\rangle\right) \neq 0 .
\end{aligned}
$$

By iterating this procedure the right number of times we obtain, from any $c \neq c^{\prime}$ such that $\Delta(c)$ is not orthogonal to $\Delta\left(c^{\prime}\right)$, a pair $d \neq d^{\prime}$ such that $\Delta(d)$ is not orthogonal to $\Delta\left(d^{\prime}\right)$ and with both idom $(d)$ and idom $\left(d^{\prime}\right)$ included in an interval of size less or equal to $s$. By shift-invariance it is therefore sufficient to check that the columns $\left\{\Delta|c\rangle \mid c \in \mathcal{C}_{f}^{I} \wedge \operatorname{idom}(c) \leq s\right\}$ are pairwise orthogonal. 


\section{A.2 Proof of Proposotion 2}

Let $I$ stand for the interval $[0, s]$ and $J$ stand for the interval $[-s, s]$.

$[\Rightarrow]$ Corollary 1 implies that the columns $\left\{\Delta|c\rangle \mid c \in \mathcal{C}_{f}\right\}$ are orthogonal if and only if we have $\forall c, c^{\prime} \in \mathcal{C}_{f}^{J} \quad\left|\left(\Delta|c\rangle, \Delta\left|c^{\prime}\right\rangle\right)\right|^{2}$ strictly positive when $c$ equals $c^{\prime}$ and zero otherwise. :

$$
\begin{aligned}
& \Leftrightarrow \prod_{i \in \operatorname{extidom}(J)}\left(\delta\left|c_{i+N}\right\rangle\right)^{\dagger}\left(\delta\left|c_{i+N}^{\prime}\right\rangle\right) \underset{c=c^{\prime}}{>} / \underset{c \neq c^{\prime}}{=0} 0 \\
& \Leftrightarrow \prod_{i \in \operatorname{extidom}(J)}\left(A\left|c_{i+\tilde{N}}, c_{i+1+\tilde{N}}\right\rangle\right)^{\dagger}\left(A\left|c_{i+\tilde{N}}^{\prime}, c_{i+1+\tilde{N}}^{\prime}\right\rangle\right) \underset{c=c^{\prime}}{\geq} / \underset{c \neq c^{\prime}}{=0} 0 \\
& \Leftrightarrow \prod_{i \in \operatorname{extidom}(J)}\left\langle c_{i+1+\tilde{N}} c_{i+1+\tilde{N}}^{\prime}|M| c_{i+\tilde{N}} c_{i+\tilde{N}}^{\prime}\right\rangle \underset{c=c^{\prime}}{>} / \underset{c \neq c^{\prime}}{=} 0 \\
& \Leftrightarrow \prod_{i \in \operatorname{extidom}(J)} M_{c_{i+1+\tilde{N}} c_{i+1+\tilde{N}, c_{i+\tilde{N}} c^{\prime}}^{\prime}} \underset{c=c^{\prime}}{ } / \underset{c \neq c^{\prime}}{=0}
\end{aligned}
$$

Summing this last equation over those configurations $c, c^{\prime} \in \mathcal{C}_{f}^{J}$ which verify $c_{0+\tilde{N}}=x, c_{0+\tilde{N}}^{\prime}=x^{\prime}$ yields, for any fixed $x, x^{\prime} \in \Sigma^{n-1}$ :

$$
\left\langle q^{n-1} q^{n-1}\left|M^{s}\right| x x^{\prime}\right\rangle\left\langle x x^{\prime}\left|M^{s}\right| q^{n-1} q^{n-1}\right\rangle \underset{x=x^{\prime}}{>} / \underset{x \neq x^{\prime}}{=} 0
$$

$[\Leftarrow]$ Consider two configurations $d, d^{\prime} \in \mathcal{C}_{f}^{I}$. By merely shifting to the center any difference between $d$ and $d^{\prime}$ one can always construct $c, c^{\prime} \in \mathcal{C}_{f}^{J}$ such that $c_{0+\tilde{N}}=$ $x, c_{0+\tilde{N}}^{\prime}=x^{\prime}$, and $\left[x=x^{\prime}\right.$ if and only if $\left.d=d^{\prime}\right]$. Now consider condition (11):

$$
\begin{aligned}
& M_{q^{n-1} q^{n-1}, \ldots} \prod_{i} M_{c_{i+\tilde{N}} c_{i+\tilde{N}}^{\prime}, c_{i+1+\tilde{N}}} c_{i+1+\tilde{N}}^{\prime} M_{\ldots, x x^{\prime}} \\
& \cdot M_{x x^{\prime}, \ldots} \prod_{i} M_{c_{i+\tilde{N}} c_{i+\tilde{N}}^{\prime}, c_{i+1+\tilde{N}} c_{i+1+\tilde{N}}^{\prime}} M_{\ldots, q^{n-1} q^{n-1}} \underset{x=x^{\prime}}{>} / \underset{x \neq x^{\prime}}{=} 0 \\
& \Leftrightarrow \prod_{i \in \operatorname{extidom}(J)}\left(\delta\left|c_{i+N}\right\rangle\right)^{\dagger}\left(\delta\left|c_{i+N}^{\prime}\right\rangle\right) \underset{c=c^{\prime}}{>} / \underset{c \neq c^{\prime}}{=} 0 \\
& \Leftrightarrow \\
& \quad \Leftrightarrow \prod_{i \in \operatorname{extidom}(I)}\left(\delta\left|d_{i+N}\right\rangle\right)^{\dagger}\left(\delta\left|d_{i+N}^{\prime}\right\rangle\right) \underset{d=d^{\prime}}{>} / \underset{d \neq d^{\prime}}{=0} 0
\end{aligned}
$$

In other words the columns $\left\{\Delta|d\rangle \mid d \in \mathcal{C}_{f}^{I}\right\}$ are orthogonal, and so Corollary 1 implies that the columns $\left\{\Delta|c\rangle \mid c \in \mathcal{C}_{f}\right\}$ are orthogonal. 


\section{A.3 Proof of Proposition 3}

$$
\begin{aligned}
\Delta & =\sum_{r, c \in \mathcal{C}_{f}}|r\rangle\langle r|\Delta| c\rangle\langle c| \\
& =\sum_{r, c \in \mathcal{C}_{f}}|r\rangle\left\langle r\left|\bigotimes_{i \in \mathbb{Z}} \delta\right| c_{i} c_{i+1}\right\rangle\langle c| \\
\langle r| \Delta & =\sum_{c \in \mathcal{C}_{f}} \prod_{i \in \mathbb{Z}}\left\langle r_{i}|\delta| c_{i} c_{i+1}\right\rangle\langle c| \\
\left\langle r\left|\Delta \Delta^{\dagger}\right| r\right\rangle & =\sum_{c \in \mathcal{C}_{f}} \prod_{i \in \mathbb{Z}}\left|\left\langle r_{i}|\delta| c_{i} c_{i+1}\right\rangle\right|^{2} \\
\| \Delta^{\dagger}|r\rangle \|^{2} & =\sum_{c \in \mathcal{C}_{f}} \prod_{i \in \mathbb{Z}} N_{c_{i} c_{i+1}}^{\left(r_{i}\right)}
\end{aligned}
$$

Say $r$ has an interval domain included in $I=[k, l]$. Then $\| \Delta^{\dagger}|r\rangle \|^{2}$ equals

$$
\sum_{c \in \mathcal{C}_{f}} \prod_{i \in-\infty \ldots k-1} N_{c_{i} c_{i+1}}^{(q)} \prod_{i \in k \ldots l} N_{c_{i} c_{i+1}}^{\left(r_{i}\right)} \prod_{i \in l+1 \ldots \infty} N_{c_{i} c_{i+1}}^{(q)}
$$

If we now restrict our sum to antecedents whose interval domain is included in $J=[k-h, l+h+1]$, each term in the sum takes the form

$$
\begin{aligned}
& N_{q q}^{(q)} \cdots N_{q c_{k-h}}^{(q)} N_{c_{k-1} c_{k}}^{(q)}\left(\prod_{i \in k \ldots l} N_{c_{i} c_{i+1}}^{\left(r_{i}\right)}\right) N_{c_{l+1} c_{i+2}}^{(q)} N_{c_{l+h+1} q}^{(q)} \cdots N_{q q}^{(q)} \\
& =N_{q c_{k-h}}^{(q)} \cdots N_{c_{k-1} c_{k}}^{(q)}\left(\prod_{i \in k \ldots l} N_{c_{i} c_{i+1}}^{\left(r_{i}\right)}\right) N_{c_{l+1} c_{l+2}}^{(q)} \cdots N_{c_{l+h+1} q}^{(q)}
\end{aligned}
$$

using $N_{q q}^{(q)}=1$. Performing the sum yields

$$
\begin{aligned}
& \left\langle q\left|\left(\prod_{k-1-h \ldots k-1} N^{(q)}\right)\left(\prod_{i \in k \ldots l} N^{\left(r_{i}\right)}\right)\left(\prod_{l+1 \ldots l+h+1} N^{(q)}\right)\right| q\right\rangle \\
& =\left\langle q\left|N^{(q)^{h}}\left(\prod_{i \in k \ldots l} N^{\left(r_{i}\right)}\right) N^{(q)}{ }^{h}\right| q\right\rangle
\end{aligned}
$$

The limit of this expression as $h$ tends to infinity remains finite. Indeed, let us suppose that the contrary is true. This implies that the row $\Delta^{\dagger}|r\rangle$ has infinite norm. But by lemma 1 this contradicts the fact that $\Delta$ is assumed to be normpreserving.

\section{A.4 Proof of Proposition 4}

By lemma 2 requiring that the rows be of unit norm of rows is equivalent to the condition that for all $t$ and $J=[-t, t]$,

$$
\sum_{r \in \mathcal{C}_{f}^{J}} \| \Delta^{\dagger}|r\rangle \|^{2}=|q \Sigma|^{2 t+1}
$$


Using proposition 3 the equation becomes

$$
\begin{aligned}
& \lim _{h \rightarrow \infty}\left\langle q\left|N^{h}\left(\sum_{r \in \mathcal{C}_{f}^{J}} \prod_{i \in J} N^{\left(r_{i}\right)}\right) N^{h}\right| q\right\rangle=|q \Sigma|^{2 t+1} \\
& \Leftrightarrow \lim _{h \rightarrow \infty}\left\langle q\left|N^{h}\left(\prod_{-t \ldots t} \sum_{\sigma \in q \Sigma} N^{(\sigma)}\right) N^{h}\right| q\right\rangle=|q \Sigma|^{2 t+1} \\
& \Leftrightarrow \lim _{h \rightarrow \infty}\left\langle q\left|N^{h} O^{2 t+1} N^{h}\right| q\right\rangle=|q \Sigma|^{2 t+1} \\
& \text { since } \sum_{\sigma \in q \Sigma} N_{x y}^{(\sigma)}=\sum_{\sigma \in q \Sigma}|\langle\sigma|\delta| x y\rangle|^{2}=1
\end{aligned}
$$

by the normalization condition.

Since $O^{2 t+1}=|q \Sigma|^{2 t} . O$ we have our condition.

\section{A.5 Proof of Proposition 5}

Since $\lim _{h \rightarrow \infty}\left\langle q\left|N^{h} O N^{h}\right| q\right\rangle=\sum_{r \in \mathcal{C}_{f}^{[0,0]}} \| \Delta^{\dagger}|r\rangle \|^{2}$ this comes as a corollary of Proposition 4

\section{A.6 Proof of Proposition 6 ,}

We have that $\vec{l} \cdot \vec{r}=\vec{l}_{q}=\vec{l}_{q}=1$ since all three are by definition equal to $\lim _{h \rightarrow \infty}\left\langle q\left|N^{h}\right| q\right\rangle=\| \Delta^{\dagger}|\ldots q q q \ldots\rangle \|^{2}=1$.(That the norm of the all-quiescent row is one stems from the fact that $\langle q|\delta| q q\rangle=1$ and $\Delta$ is norm-preserving.) All entries in $\vec{l}$ and $\vec{r}$ are nonnegative because all entries in $N$ and $|q\rangle$ are nonnegative.

Now note that

$$
\begin{aligned}
\left(\sum_{i} \vec{l}_{i}\right)\left(\sum_{i} \vec{r}_{i}\right) & =\lim _{h \rightarrow \infty}\left\langle q\left|N^{h} O N^{h}\right| q\right\rangle \\
& =\sum_{r \in \mathcal{C}_{f}^{[0,0]}} \| \Delta^{\dagger}|r\rangle \|^{2} \leq|q \Sigma|
\end{aligned}
$$

where the last line was obtained using $\sum_{\sigma \in q \Sigma} N_{x y}^{(\sigma)}=1$ and Lemma 11 (It is clear that this inequality is saturated if and only if $\Delta$ has unit rows, since this was the content of proposition (4) Using this and nonnegativity we have $\left(\sum_{i} \vec{r}_{i}\right)=\vec{l}_{q}\left(\sum_{i} \vec{r}_{i}\right) \leq\left(\sum_{i} \vec{l}_{i}\right)\left(\sum_{i} \vec{r}_{i}\right) \leq|q \Sigma|$, hence the entries in $\vec{r}$ are finite, and symmetrically so for $\vec{l}$.

Say $\exists x \in \Sigma,\left[\vec{l}_{x}=0 \wedge \vec{r}_{x}=0\right]$. Then $\langle\ldots q q \ldots|\Delta| \ldots q q x q q \ldots\rangle \neq 0$, which contradicts the fact that $\langle q|\delta| q q\rangle=1$ and $\Delta$ is norm-preserving, and yields condition (iii). Say $\exists x, y \in \Sigma,\left[N_{x y} \neq 0 \wedge \vec{l}_{x}=0 \wedge \vec{l}_{y}=0\right]$. Then $\langle\ldots q q \ldots|\Delta| \ldots q q x y q q \ldots\rangle \neq 0$, which again contradicts the fact that $\langle q|\delta| q q\rangle=$ 1 and $\Delta$ is norm-preserving, and yields condition (iv). 
The fact that $\vec{r}$ is an eigenvector of $N$ is trivial, since $\vec{r}=\lim _{h \rightarrow \infty} N^{h}|q\rangle=$ $\lim _{h \rightarrow \infty} N^{h+1}|q\rangle=N \vec{r}$. We now show it is minimal in $S=\{v \mid \mathbf{0} \leq v \wedge N v=$ $\left.v \wedge v_{q}=1\right\}$. Say $w \in S$. We have $w=|q\rangle+u$, with $u$ a vector having nonnegative entries. Hence

$$
\begin{aligned}
w & =\lim _{h \rightarrow \infty} N^{h} w \\
& =\lim _{h \rightarrow \infty} N^{h}|q\rangle+\lim _{h \rightarrow \infty} N^{h} u \\
& =\vec{r}+\lim _{h \rightarrow \infty} N^{h} u .
\end{aligned}
$$

But since $N$ and $u$ have nonnegative entries, $\lim _{h \rightarrow \infty} N^{h} u$ has nonnegative entries and $\vec{r} \leq w$. Symmetrically so for $\vec{l}$.

\section{References}

1. S. Amoroso, Y. N. Pratt, Decision procedures for surjectivity and injectivity of parallel maps for tesselations structures, J. Comp. Syst. Sci., 6, 448-464, (1972).

2. P. Arrighi, C. Patricot, On Quantum Operations as Quantum States, Annals of Phys. 311, 26-52, (2004).

3. T. Boykett, Efficient exhaustive enumeration of reversible one dimensional cellular automata, Theoretical Computer Science, (2004).

4. C. Dürr, H. LêThanh, M. Santha, A decision procedure for well formed quantum cellular automata, Random Structures and Algorithms, 11, 381-394, (1997).

5. C. Dürr, M. Santha, A decision procedure for unitary quantum linear cellular automata, SIAM J. of Computing, 31(4), 1076-1089, (2002).

6. R. P. Feynman, Quantum mechanical computers, Found. Phys. 16, 507-531, (1986).

7. P. Hoyer, Note on linear quantum cellular automata, manuscript.

8. O. H. Ibarra, T.Jiang, On the computing power of one-way cellular arrays, ICALP, 550-562, (1987).

9. R. Landauer, Irreversibility and heat generation in the computing process, IBM J. Res. Dev., 5(183), (1961).

10. D. A. Meyer, Unitarity in one dimensional nonlinear quantum cellular automata, arXiv:quant-ph/9604011

11. J. Pedersen, Cellular automata as algebraic systems, Complex Systems, 6, 237-250, (1992).

12. B. Schumacher, R. F. Werner, Reversible quantum cellular automata, arXiv:quant-ph/0405174

13. W. Van Dam, Quantum Cellular Automata, Master thesis, Department of Mathematics and Computer Science, University of Nijmegen, The Netherlands, (1996).

14. J. Watrous, On one dimensional quantum cellular automata, Complex Systems 5(1), 19-30, (1991). 